\title{
Millimeter observations of radio-loud active galaxies
}

\author{
I. M. van Bemmel ${ }^{1,2}$ and F. Bertoldi ${ }^{3}$ \\ 1 European Southern Observatory, Karl-Schwarzschildstr. 2, 85748 Garching bei München, Germany \\ 2 Kapteyn Astronomical Institute, PO Box 800, 9700 AV Groningen, The Netherlands \\ 3 Max-Planck-Institut für Radioastronomie, Auf dem Hügel 69, 53121 Bonn, Germany
}

Received 9 August 2000 / 12 December 2000

\begin{abstract}
In order to study the nature of the far-infrared emission observed in radio-loud active galaxies, we have obtained $1.2 \mathrm{~mm}$ observations with the IRAM $30 \mathrm{~m}$ telescope for a sample of eight radio-loud active galaxies. In all objects we find that the $1.2 \mathrm{~mm}$ emission is dominated by non-thermal emission. An extrapolation of the non-thermal radio spectrum indicates that the contribution of synchrotron emission to the far-infrared is less than $10 \%$ in quasars, and negligible in the radio galaxies. The quasars in the sample show signs of relativistic beaming at millimeter wavelengths, and the quasar 3C 334 shows evidence for strong variability.
\end{abstract}

Key words. galaxies: active - galaxies: photometry - quasars: general - infrared: general - infrared: galaxies radio continuum: galaxies

\section{Introduction}

Photometry from radio to infrared wavelengths has shown that double-lobed radio-loud quasars show deep minima in their spectral energy distributions at millimeter wavelengths (Antonucci et al. 1990), which indicates that the radio emission arises from components which cannot be related to the infrared emission. However, the nature of the far-infrared emission observed in double-lobed radio sources remains unclear. Most evidence (Haas et al. 1998; Polletta et al. 2000, and references therein) points at a thermal nature of the infrared emission, although a possible contribution from relativistically beamed, nonthermal emission has not yet been carefully determined. Relativistic beaming can play an important role in the infrared emission of quasars, in which the relativistic jet is oriented closer to the line of sight (Barthel 1989). In radiogalaxies however, beaming is thought to have a negligible effect (Hoekstra et al. 1997).

The significance of beaming may be estimated by extrapolating the radio core spectra to infrared wavelengths. The non-thermal emission from the radio lobes can be safely neglected, as these have no bulk relativistic motions and thus show no beaming. However, the exact shape of the core spectrum is unknown at high frequencies.

In order to improve the quality of the extrapolation of the non-thermal spectrum, we measured $1.2 \mathrm{~mm}$ continuum fluxes for eight $3 \mathrm{CR}$ objects, of which seven have

Send offprint requests to: I. M. van Bemmel, e-mail: bemmel@astro.rug.nl been observed with ISOPHOT on board ISO (Lemke et al. 1996; Kessler et al. 1996) and with the NRAO Very Large Array (VLA) (van Bemmel et al. 2000). We here compare the $1.2 \mathrm{~mm}$ fluxes with the integrated radio and infrared data, and for the quasars in the sample, with their radio core spectra.

The general properties of our objects are listed in Table 1. The sample consists of three radio-loud quasars (QSR), two broad-line radio galaxies (BLRG), one narrowline radio galaxy (NLRG) and the radio structure 3C 59. The latter was previously misidentified with the Seyfert 1 galaxy RBS 0281. The maps of Meurs \& Unger (1991) show three components: a north-eastern source (the original 3C 59), a central source associated with RBS 0281, and a south-western source. 3C 59 has no optical counterpart, so it is either a lobe of RBS 0281 , or a background coredominated quasar. With ISOPHOT only RBS 0281 was observed. We shall refer to the Seyfert galaxy as RBS 0281, and to the north-eastern hotspot/background object as $3 \mathrm{C} 59$.

\section{Observations and data reduction}

\subsection{IRAM $30 \mathrm{~m}$}

Continuum observations at $1.2 \mathrm{~mm}(250 \mathrm{GHz})$ were obtained between 12 and 15 December 1999 with the MaxPlanck Millimeter Bolometer (MAMBO; Kreysa et al. 1998) at the IRAM $30 \mathrm{~m}$ telescope on Pico Veleta, Spain (Baars et al. 1987). MAMBO is a 37-element bolometer array, sensitive between 190 and $315 \mathrm{GHz}$. The half peak 
Table 1. Basic properties of the observed objects. Types are: NLRG $=$ narrow-line radio galaxy, BLRG $=$ broad line radio galaxy, QSR $=$ radio quasar, Sey $1=$ Seyfert $1, ?=$ unknown. We adopt the most common classification in the literature. $L_{178}$ assumes $H_{0}=75 \mathrm{~km} \mathrm{~s}^{-1} \mathrm{Mpc}^{-1}, q_{0}=0.5$ and $F_{\nu} \propto \nu^{-1}$. The last column lists the size of the radio structure associated with the object, only the central component size is given for RBS 0281

\begin{tabular}{lccccc}
\hline Name & IAU & $z$ & type & $\begin{array}{c}L_{178} \\
{[\mathrm{~W} / \mathrm{Hz}]}\end{array}$ & $\begin{array}{c}\text { size } \\
{\left[{ }^{\prime \prime}\right]}\end{array}$ \\
\hline 3C 33.1 & $0106+729$ & 0.181 & BLRG & 26.89 & 227 \\
3C 59 & $0204+293$ & - & $?$ & 24.01 & $\sim 4$ \\
RBS 0281 & $0204+293$ & 0.110 & Sey1 & - & $\sim 1$ \\
3C 67 & $0221+276$ & 0.310 & BLRG & 27.28 & 2.5 \\
3C 277.1 & $1250+568$ & 0.321 & QSR & 27.24 & 1.7 \\
3C 323.1 & $1545+210$ & 0.264 & QSR & 27.13 & 69 \\
3C 334 & $1618+177$ & 0.555 & QSR & 27.88 & 58 \\
3C 460 & $2318+235$ & 0.268 & NLRG & 27.08 & 8 \\
\hline
\end{tabular}

sensitivity range is $210-290 \mathrm{GHz}$, with an effective bandpass center, somewhat dependent on the spectral slope of the observed emission, at $250 \mathrm{GHz}$. The beam for the feed horn of each bolometer is matched to the telescope beam of $10.6^{\prime \prime}$, and the bolometers are arranged in a hexagonal pattern with a beam separation of $22^{\prime \prime}$. Observations were made in standard on-off mode, with $2 \mathrm{~Hz}$ chopping of the secondary reflector by $32^{\prime \prime}$. The pointing accuracy is typically $2^{\prime \prime}$. The target was centered on the central bolometer of the array, and after each $10 \mathrm{~s}$ of integration, the telescope was nodded so that the previous "off" beam becomes the "on" beam. Each scan of twelve 10 s subscans lasts $3 \mathrm{~min}$, of which $1 \mathrm{~min}$ integration falls on the sources, $1 \mathrm{~min}$ off source, and $1 \mathrm{~min}$ is used to move the telescope and start the integration. Typically 15 to 20 of such scans were performed for each object.

Gain calibration was performed using observations of Mars, Uranus, and Ceres, resulting in a flux calibration factor of 12500 counts per Jansky, which we estimate to be accurate to $15 \%$. A sky opacity correction factor was measured every 2 hours through total power sky dips.

The data were analyzed using the MOPSI software package (Zylka 1998). For each bolometer the temporally correlated variation of the sky signal (sky-noise) was computed using the signals of neighbouring bolometers. The correlated noise is iteratively determined for each channel and subtracted.

Due to unintentional mispointing, for 3C 59 and RBS 0281 the targeted positions differ from the radio positions in the maps of Meurs \& Unger (1991, see also Table 2). A signal is measured at the targeted position toward RBS 0281, which however is $10^{\prime \prime}$ off the radio peak, so that the millimeter flux from the peak radio position could well be higher than observed. Toward 3C 59, a 7 mJy signal was picked up by an off-center channel, closely corresponding to the position of component D in the Meurs
Table 2. Observed positions for 3C 59 and RBS 0281: central channel position and the peak flux positions in the Meurs \& Unger (1991) maps are given. For 3C 59 we also list the position at which the signal is detected

\begin{tabular}{llll}
\hline Object & Position of & RA (2000) & DEC (2000) \\
\hline 3C 59 & MAMBO target & 020709.6 & 293124 \\
& radio peak & 020710.1 & 293145.1 \\
& detection & 020709.7 & 293144 \\
\hline \multirow{2}{*}{ RBS 0281 } & MAMBO target & 020702.3 & 293055.1 \\
& radio peak & 020702.2 & 293046.8 \\
\hline
\end{tabular}

\& Under radio map. Since the chances of observing a background source are very small, we assume that the off-center channel detects 3C 59, but also here the peak flux could be higher than the observed flux. Since the MAMBO fluxes toward RBS 0281 and 3C 59 are uncertain, we will not make use of them in the analysis. They will be re-measured in a future observing campaign.

\subsection{Radio and infrared data}

We collected radio fluxes for our objects from the NASA/IPAC Extragalactic Database (NED). Where available, we adopt those given at $178 \mathrm{MHz}, 1.4 \mathrm{GHz}$, $2.7 \mathrm{GHz}$, and $4.9 \mathrm{GHz}$. For the three quasars additional fluxes from the unresolved core are available at 4.9, 15, 25 and $43 \mathrm{GHz}$ from a previous programme (van Bemmel et al. 2000, hereafter BBG). For RBS 0281 only one radio point was found.

The infrared data were obtained with ISOPHOT, using the P1, P2, C100 and C200 detectors in raster-mapping mode. A detailed description of the data reduction is given in BBG, including a list of the resulting flux densities. ISOPHOT data are available at 60,90 and $160 \mu \mathrm{m}$ for all objects except 3C 59. For 3C 33.1 and RBS 0281 we have additional ISOPHOT data at 12 and $25 \mu \mathrm{m}$. For 3C 323.1 an upper limit at $10 \mu \mathrm{m}$ was obtained by Rieke \& Low (1972).

\section{Results}

Table 3 lists the radio and millimeter flux densities. The radio fluxes are integrated fluxes, which include the lobes and other extended structures. The millimeter fluxes only include the part of the object within the IRAM $30 \mathrm{~m}$ telescope beam. The resulting spectral energy distributions (SED) are plotted in Fig. 1, along with the radio flux densities of the unresolved core emission for the quasars.

The $1.2 \mathrm{~mm}$ flux densities are always lower than the infrared and the integrated radio flux densities, consistent with observations by Antonucci et al. (1990). They found that quasars show a minimum in their SEDs at millimeter wavelengths. We obtained similar results for radio galaxies. The millimeter flux densities are even lower than the fluxes expected from an extrapolation of the integrated radio spectra, which we show as dashed lines in Fig. 1. For 
Table 3. NED archive radio fluxes $S_{\nu}$ and newly obtained MAMBO $250 \mathrm{GHz}$ fluxes for our sample. Additional data can be found in van Bemmel et al. (2000). Brackets indicate an off-center detection

\begin{tabular}{lccccc}
\hline Name & $\begin{array}{c}178 \mathrm{MHz} \\
(\mathrm{Jy})\end{array}$ & $\begin{array}{c}1.4 \mathrm{GHz} \\
(\mathrm{Jy})\end{array}$ & $\begin{array}{c}2.7 \mathrm{GHz} \\
(\mathrm{Jy})\end{array}$ & $\begin{array}{c}4.9 \mathrm{GHz} \\
(\mathrm{mJy})\end{array}$ & $\begin{array}{c}250 \mathrm{GHz} \\
(\mathrm{mJy})\end{array}$ \\
\hline 3C 33.1 & $11.4 \pm 0.9$ & - & - & $820 \pm 62$ & $6 \pm 2$ \\
3C 59 & $5.3 \pm 1.0$ & $1.60 \pm 0.03$ & - & $670 \pm 87$ & $(7 \pm 1)$ \\
RBS 0281 & - & $0.026 \pm 0.002$ & - & - & $(>3)$ \\
3C 67 & $9.0 \pm 1.1$ & $3.10 \pm 0.09$ & - & $860 \pm 113$ & $10.3 \pm 0.7$ \\
3C 277.1 & $8.9 \pm 0.9$ & $2.50 \pm 0.09$ & $1.56 \pm 0.06$ & $880 \pm 90$ & $20.6 \pm 1.5$ \\
3C 323.1 & $10.0 \pm 1.3$ & $2.45 \pm 0.12$ & $1.50 \pm 0.08$ & $840 \pm 112$ & $13.5 \pm 0.9$ \\
3C 334 & $10.9 \pm 0.8$ & $2.20 \pm 0.11$ & $1.00 \pm 0.05$ & $500 \pm 76$ & $<4$ \\
3C 460 & $8.1 \pm 1.0$ & $1.60 \pm 0.08$ & $0.90 \pm 0.05$ & $440 \pm 59$ & $4.3 \pm 1.2$ \\
\hline
\end{tabular}

this extrapolation we fitted a power law to the radio data with $\nu>1 \mathrm{GHz}$. In cases where a flux was available at only one frequency, we use the average slope of the other objects, excluding 3C 59 and RBS 0281 though.

Half of the objects in our sample have radio sizes larger than the $10.6^{\prime \prime}$ beam of the IRAM $30 \mathrm{~m}$ telescope at $1.2 \mathrm{~mm}$. In these cases a direct comparison with the radio data may not be meaningful, unless we assume that the radio lobes are not contributing to the millimeter emission (see Sect. 4.3). For the quasars, core radio fluxes are available at even higher resolution $\left(1-2^{\prime \prime}\right)$. The core radio SED shows no obvious relation to the millimeter fluxes, except maybe for 3C 323.1, where the core and millimeter fluxes show a typical self-absorbed synchrotron spectrum.

To test whether the millimeter flux could arise from a cold, thermal component that also gives rise to the $160 \mu \mathrm{m}$ emission, we fit an optically thin grey-body spectrum to the far-infrared flux, using a dust emissivity index $\beta=1.6$. This value is an average of observed values in nearby active and normal galaxies. To be conservative, we adopted dust temperatures of $20 \mathrm{~K}$, which is even colder than the coldest dust found in active galaxies with ISO (e.g. Siebenmorgen et al. 1999). The temperature is typical for dust in Galactic clouds and cirrus. The dust masses implied by such a cold component range from a few $10^{7}$ to several $10^{8} M_{\odot}$. From the extrapolated grey-body spectra it appears that the millimeter flux is dominated by non-thermal emission. In 3C 460, e.g., the $1.2 \mathrm{~mm}$ flux could be dominated by thermal dust emission if $\beta<1.4$ or $T<16$, but then the implied dust mass would be larger than $510^{8} M_{\odot}$. Such a large dust mass is hard to reconcile with 3C 460 being an elliptical galaxy. There is only circumstantial evidence for a very cold dust component $(T<15 \mathrm{~K})$ in galaxies. Starbursts and active galaxies do not show very cold dust emission at millimeter wavelengths.

3C 334 is probably a variable millimeter source. Previous observations with the Owens Valley Radio Observatory found a $3 \mathrm{~mm}$ flux density of $37 \pm 2 \mathrm{mJy}$ (van Bemmel et al. 1998). Assuming $F_{\nu} \propto \nu^{-1}$, a $1.2 \mathrm{~mm}$ flux of $15 \mathrm{mJy}$ would be expected, much higher than our upper limit of $3 \mathrm{mJy}$. 3C 334 is known to be variable at
Table 4. Monochromatic $250 \mathrm{GHz}$ luminosities (in $\mathrm{W} \mathrm{Hz}^{-1}$ ) and radio spectral indices. Brackets indicate an off-center detection. $\left({ }^{*}\right)$ for RBS $0281 \alpha_{250}^{1.4}$ is given instead of $\alpha_{250}^{4.9}$

\begin{tabular}{lcccc}
\hline Name & $\log L_{250}$ & $\alpha_{1.4}^{178}$ & $\alpha_{4.9}^{1.4}$ & $\alpha_{250}^{4.9}$ \\
\hline 3C 33.1 & 23.61 & - & - & -1.28 \\
3C 59 & - & -0.61 & -0.64 & $(-1.18)$ \\
RBS 0281 & $(>22.86)$ & - & - & $(>-0.42)^{*}$ \\
3C 67 & 24.33 & -0.52 & -1.02 & -1.15 \\
3C 277.1 & 24.67 & -0.62 & -0.83 & -0.97 \\
3C 323.1 & 24.30 & -0.68 & -0.85 & -1.07 \\
3C 334 & $<24.46$ & -0.78 & -1.18 & $<-1.25$ \\
3C 460 & 23.82 & -0.79 & -1.03 & -1.2 \\
\hline QSR average & 24.49 & -0.69 & -0.96 & -1.02 \\
RG average & 23.92 & -0.65 & -1.03 & -1.21 \\
\hline
\end{tabular}

$4.85 \mathrm{GHz}$, with variations of order $10 \%$ over a few decades (van Bemmel et al. 1998). Variability tends to increase at higher frequencies, but one would not expect a dramatic change in flux such as implied by the OVRO and MAMBO measurements.

\subsection{Luminosities and spectral indices}

Adopting $H_{0}=75, q_{0}=0.5$, and $k \Lambda=0$, we computed luminosity densities in order to compare the millimeter luminosities of quasars and radio galaxies (Table 4). 3C 59 is excluded, because its redshift has not yet been determined. Although we find that the infrared luminosities are comparable between the classes, there is evidence that the quasars are brighter at $1.2 \mathrm{~mm}$ than the radio galaxies. At $178 \mathrm{MHz}$ the quasars are only marginally brighter.

The sample is too small to draw firm conclusions, but it appears that the stronger millimeter emission from quasars may be due to a beamed component. When comparing the integrated flux at $178 \mathrm{MHz}$ and $1.4 \mathrm{GHz}$, the quasars are a factor 1.5 brighter than the radio galaxies. In case of isotropic synchrotron emission over the whole radio spectrum, this factor should be constant. However, it appears to increase toward higher frequencies; at $250 \mathrm{GHz}$ 


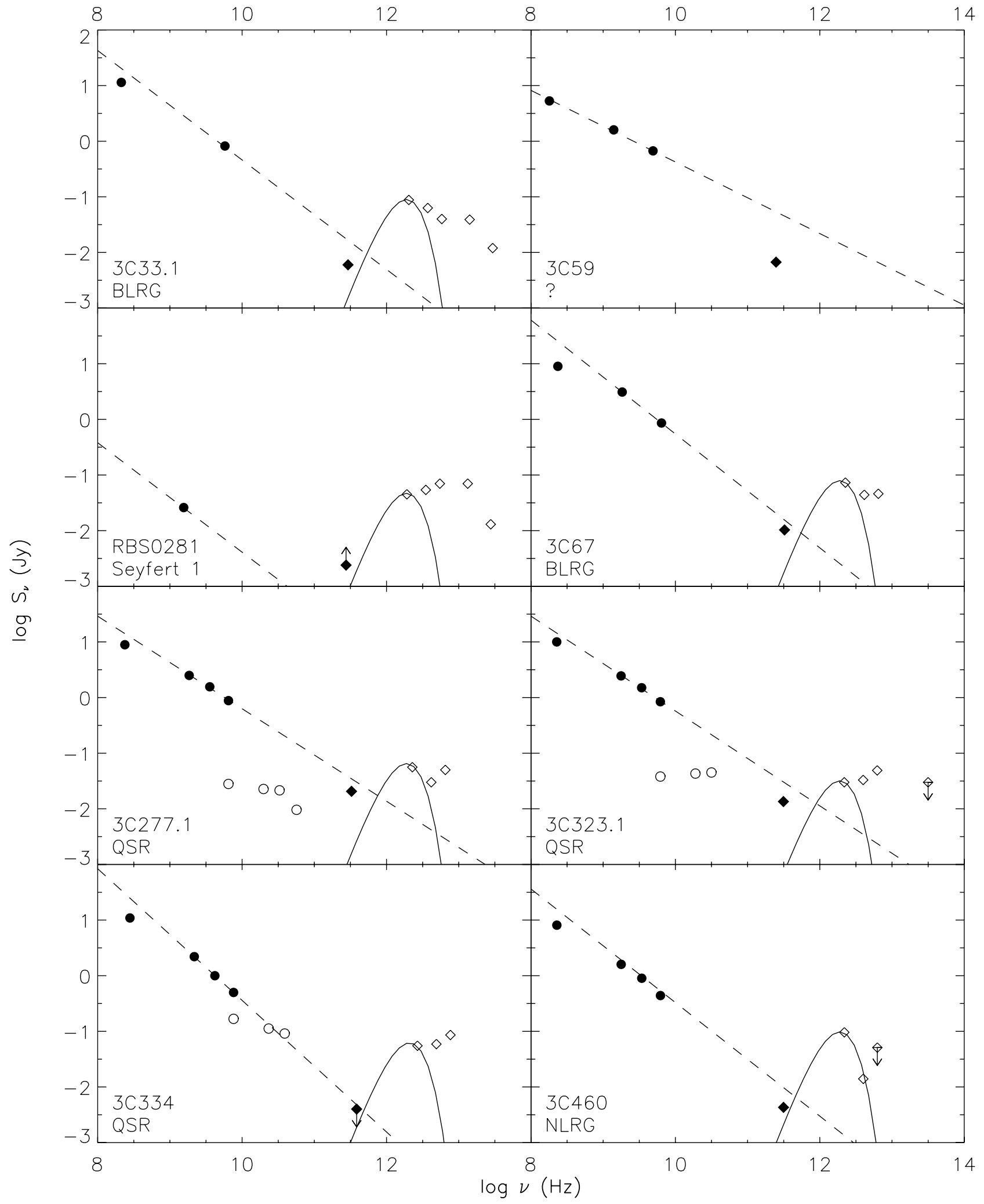

Fig. 1. Rest frame spectral energy distributions of the objects observed with MAMBO. For 3C 59 we assume $z=0$. Filled circles show the total integrated radio fluxes, open circles show the unresolved core radio fluxes, the filled diamond the MAMBO $1.2 \mathrm{~mm}$ fluxes, and open diamonds are ISOPHOT fluxes. The solid lines represent grey-body $T=20 \mathrm{~K}$ spectra, matching the $160 \mu \mathrm{m}$ fluxes. The dashed lines are power law fits to $>1 \mathrm{GHz}$ integrated radio data 
the quasars are about three times brighter than the radio galaxies. This indicates that we observe an additional component, which is unlikely to be due to dust emission, since that would be optically thin and therefore also visible in the radio galaxies. For synchrotron emission, the excess can only be beamed emission, which causes a natural anisotropy. It would be desirable to confirm this trend with a larger sample.

There is clear evidence of spectral steepening of the radio spectrum towards millimeter wavelengths. The average spectral index for the entire sample ranges from -0.7 at the lowest frequency to -1.2 at millimeter wavelengths (Table 4). Spectral steepening occurs in most objects in our sample, irrespective of their size or the resolution of the observations.

\section{Discussion}

\subsection{Nature of the millimeter and far-infrared emission}

We find that the $1.2 \mathrm{~mm}$ flux densities are well below those expected from an extrapolation of the total radio flux densities. If cold dust gives rise to the millimeter emission, then the millimeter flux densities are expected to lie above the extrapolated radio spectra, and the grey-body extrapolation should fit the observed flux. In our data the opposite is true, and therefore the millimeter emission is probably dominated by synchrotron emission, except maybe for 3C 460, where this case is not so clear.

Since the far-infrared fluxes are more than an order of magnitude higher than the millimeter fluxes, the farinfrared emission is likely to arise from dust. The clear minimum of the SED at millimeter wavelengths excludes that the far-infrared emission is the continuation of the radio synchrotron spectrum of the radio lobes or the core.

If the far-infrared emission were due to synchrotron radiation, it would have to arise from a very young electron population. The younger the electrons, the closer they must be to the core, so that their emission would be even more strongly beamed than synchrotron emission at millimeter wavelengths. As a consequence, the quasars should be brighter than radio galaxies at infrared wavelengths, for which there is no evidence in our data. Instead we find that in the infrared the quasars and radio galaxies are comparably bright, while at $1.2 \mathrm{~mm}$ the quasars are clearly brighter than the radio galaxies.

The possible contribution of relativistically beamed synchrotron emission to the far-infrared emission is estimated by extrapolating the radio SED. This yields on average a less than $10 \%$ contribution in quasars, and a less than $1 \%$ contribution in radio galaxies. Although in 3C 323.1 the core radio spectrum would extrapolate well to the observed far-infrared fluxes, the $1.2 \mathrm{~mm}$ emission falls below this extrapolation, indicating a turnover in the core spectrum, which excludes a significant contribution of non-thermal emission to the far-infrared.
An earlier study of the infrared emission from radioloud active galaxies indicated that quasars are significantly brighter at $60 \mu \mathrm{m}$ than radio galaxies (Heckman et al. 1992). This does not contradict what we find from our sample. The objects in the Heckman sample have an average redshift $\sim 0.5-0.9$, thus in their rest frame the emission emerges at $\sim 30-40 \mu \mathrm{m}$. If the infrared emission arises from a circumnuclear torus, the dust is likely to be optically thick up to $60 \mu \mathrm{m}$ (Pier \& Krolik 1992; Granato \& Danese 1994), and the emerging flux depends on the orientation of the torus. According to these models, the observed flux could vary by orders of magnitude, depending on the torus' optical depth. However, ISOPHOT studies (BBG) show no conclusive evidence that quasars are intrinsically brighter in the far-infrared than radio galaxies, when they are matched in radio power and redshift.

\subsection{Relativistic beaming in quasars}

Unified models for radio-loud AGN (Urry \& Padovani 1995; Barthel 1989) suggest that quasars are oriented with their jets closer to the line of sight than radio galaxies. This implies that any beamed component is more evident in quasars, but also that isotropic emission, such as optically thin dust emission, should not differ among the types. Our observations confirm this picture, showing that quasars are more luminous at millimeter wavelengths, where we expect relativistic beaming, whereas they do not differ in their far-infrared luminosity, which is due to isotropic cold dust emission, and therefore unaffected by beaming. The luminosity ratio QSR/RG increases with frequency, which can only naturally be explained by beaming.

If this trend is confirmed in larger samples, millimeter observations in combination with radio observations could provide a direct measure of the orientation of a source. E.g. for a quasar and a radio galaxy of comparable $178 \mathrm{MHz}$ power, the radio galaxy provides the unbeamed radio SED that can be subtracted from the quasar SED. The remaining emission is then due to beaming, and the strength of this beamed component depends directly on the viewing angle of the source.

\subsection{Hotspot and lobe emission}

For three of our objects, the radio structures are larger than the beam size of the IRAM $30 \mathrm{~m}$ telescope, so that the hotspots fall outside the central bolometer channel. We made no attempt to observe the hotspots separately.

The radio fluxes for all objects are integrated fluxes, including the lobe emission. The MAMBO fluxes are also integrated fluxes for the small objects, but core fluxes for the objects with larger radio sizes than the IRAM beam. If the millimeter emission would be dominated by the lobe emission in all objects, there should be a clear turnover in the SEDs of the large objects, where the lobes are not observed with MAMBO. On the other hand, if the core 
dominates the millimeter emission in all objects, the SEDs should be comparable, irrespective of object radio size. We observe no clear difference between the radio-millimeter SED of large and small objects, which seems to indicate that the core is dominating the millimeter emission. This does not imply that the lobes do not emit any millimeter emission, e.g. in Cygnus A the hotspots have been clearly detected with SCUBA at $850 \mu \mathrm{m}$ (Robson et al. 1998).

However, there might be a relation between the luminosity density of the lobes at $1.2 \mathrm{~mm}$ and the size of the radio structure. Small radio sources are known to have much flatter spectra (Murgia et al. 1999) and thus can have much stronger lobes. In our MAMBO observations, all small sources are unresolved and thus we cannot tell which component is dominating. If the lobes dominate in small objects and the core dominates in larger ones, the expected difference in spectral indices will not be visible. We always observe the dominating regions, i.e. the lobes in the small radio sources and only the core in the larger ones. Judging from the radio core fluxes in 3C 277.1, the core is not the dominant $1.2 \mathrm{~mm}$ source here. The same can be true for 3C 67 and $3 \mathrm{C} 460$, which are also small radio sources. The lobe emission will dilute the amount of beaming observed in small objects, thus for a proper estimate of the amount of beaming the objects should have comparable radio sizes and be larger than the IRAM $30 \mathrm{~m}$ telescope beam.

\subsection{Variability}

We find evidence for variability in 3C 334. Since we have only one observation for each object, variability cannot be ruled out for the other objects. Previous SCUBA observations of the other quasars (BBG) are consistent with the MAMBO detections, confirming the spectral steepening and the thermal nature of the infrared emission. For the radio galaxies there is remarkably little dispersion in the observed spectral slopes. Thus, variability must be small in all other objects.

\section{Conclusions}

The main conclusions, drawn from our continuum observations at $1.2 \mathrm{~mm}$ of eight radio-loud active galaxies, can be summarized as follows:

- The millimeter emission is dominated by non-thermal processes, whereas the far-infrared emission must be thermal;

- Any thermal contribution to the millimeter emission arising from cold dust is estimated to be less than $15 \%$, except for 3C 460, where the case is not clear;

- An extrapolation of the non-thermal radio spectrum to far-infrared wavelengths shows that the contribution of non-thermal emission at $160 \mu \mathrm{m}$ is less than $10 \%$;

- There is evidence that at $1.2 \mathrm{~mm}$ the quasar emission is stronger, and therefore possibly more beamed, than that from radio galaxies;
- The far-infrared luminosity on the other hand, does not differ between quasars and radio galaxies. Most likely because it arises from optically thin thermal emission, but optical thickness effects can play a role up to $100 \mu \mathrm{m}$ in the rest frame of the objects;

- Radio and millimeter observations provide an interesting test for unification models of radio-loud active galaxies, in that they can provide a measure of the amount of beamed emission. Millimeter observations, in combination with radio observations, of a larger sample of radio-loud active galaxies are needed to verify this.

Acknowledgements. Thanks to Bob Fosbury and Peter Barthel for their motivation and help on the manuscript. Thanks to Alessandra Bertarini for assisting with the observations, and to the referee, Neal Jackson, for comments which greatly improved the manuscript. Special thanks to the MPIfR bolometer team for providing MAMBO and support, and to R. Zylka for writing the MOPSI data reduction package.

NED is operated by the Jet Propulsion Laboratory, California Institute of Technology, under contract with the National Aeronautics and Space Administration. ISO is an ESA project with instruments funded by ESA Member States (especially the PI countries: France, Germany, The Netherlands and the UK) and with the participation of ISAS and NASA.

\section{References}

Antonucci, R., Barvainis, R., \& Alloin, D. 1990, ApJ, 353, 416 Baars, J. W. M., Hooghoudt, B. G., Mezger, P. G., \& de Jonge, M. J. 1987, A\&A, 175, 319

Barthel, P. D. 1989, ApJ, 336, 606

Granato, G. L., \& Danese, L. 1994, MNRAS, 268, 235

Heckman, T. M., Chambers, K. C., \& Postman, M. 1992, ApJ, 391,39

Hoekstra, H., Barthel, P .D., \& Hes, R. 1997, A\&A, 319, 757

Kessler, M. F., Steinz, J. A., Anderegg, M. E., et al. 1996, A\&A, 315, L27

Kreysa, E., Gemuend, H.-P., Gromke, J., et al. 1998, SPIE, 3357,319

Lemke, D., Klaas, U., Abolins, J., et al. 1996, A\&A, 315, L64

Meurs, E. J. A., \& Unger, S. W. 1991, A\&A, 252, 63

Murgia, M., Fanti, C., Fanti, R., et al. 1999, A\&A, 345, 769

Pier, E. A., \& Krolik, J. H. 1992, ApJ, 401, 99

Polletta, M., Courvoisier, T. J.-L., Hooper, E. J., \& Wilkes, B. J. 2000, A\&A, 362, 75

Rieke, G. H., \& Low, F. J. 1972, ApJ, 176, L95

Robson, E. I., Leeuw, L. L., Stevens, J. A., \& Holland, W. S. 1998, MNRAS, 301, 935

Siebenmorgen, R., Krügel, E., \& Chini, R. 1999, A\&A, 351, 495

Urry, C. M., \& Padovani, P. 1995, PASP, 107, 803

van Bemmel, I. M., Barthel, P. D., \& Yun, M. S. 1998, A\&A, 334, 799

van Bemmel, I. M., Barthel, P. D., \& de Graauw, T. (BBG) 2000, A\&A, 359, 523

Zylka, R. 1998, Pocket Cookbook for MOSPI Software, http://www.iram.es/Telescope/manuals/Datared/ pockroo.ps 